\title{
Uma Aplicação Híbrida de Sistemas Neuro-Fuzzy combinado com Análise de Sensibilidade para a Estimativa de Parâmetros em Problemas Inversos em Transferência Radiativa
}

\author{
Mauro C. C. Gil \\ Centro Universitário Estadual da Zona Oeste - UEZO \\ 23070-200 - Rio de Janeiro, RJ \\ Email: maurogil@uezo.rj.gov.br \\ Antônio J. da Silva Neto, Luiz Biondi Neto \\ Universidade do Estado do Rio de Janeiro - Instituto Politécnico \\ 28630-050 - Nova Friburgo, RJ \\ Email: ajsneto@iprj.uerj.br_luizbiondi@terra.com.br
}

\begin{abstract}
Resumo: Problemas inversos são muito suscetíveis a erros, devido aos ruídos que estão sempre presentes em dados experimentais. Portanto, o tratamento é essencial na formulação e solução de tais problemas. Assim, um sistema de inferência que combina uma medida dessa ambiguidade é necessário e seja compreensivel e apropriadamente interpretada pelo usuário. Neste contexto, a lógica fuzzy, que se baseia na capacidade do ser humano de lidar com dados imprecisos e vagos, oferece uma maneira de lidar com este problema. Neste trabalho tal abordagem é aplicada em conjunto com redes neurais artificiais em sistemas neuro-fuzzy híbridos, na solução de um problema de transferência radiativa inverso. Propõe-se uma metodologia para estimar as propriedades radiativas (o albedo simples, a espessura óptica e as refletividades difusas), a partir dos valores das intensidades de radiação que sai do meio participante, usando uma abordagem híbrida de sistemas neuro-fuzzy, que combina o uso de sistemas de inferência fuzzy com redes neurais artificiais. Observou-se que a solução com sistemas neuro-fuzzy, conduz a resultados de baixa qualidade, quando os dados com baixa sensibilidade são utilizados. Uma análise de sensibilidade foi utilizada e o sistema neuro-fuzzy híbrido foi então construído utilizando os dados com maiores sensibilidades. Uma melhoria significativa foi então obtida a partir de diferentes casos de teste na solução deste problema.
\end{abstract}

\section{Introdução}

A solução de problemas diretos e inversos de Transferência Radiativa é uma ferramenta eficiente para a estimativa de propriedades ópticas em diferentes meios. A análise de problemas inversos envolvendo a interação de diversos tipos de partículas e radiação, tais como nêutrons, raios gama e fótons, com meios participantes, ou seja, meios absorvedores, emissores e espalhadores, tem sido bastante utilizada no desenvolvimento de técnicas para aplicações em engenharia, medicina, geofísica, astrofísica, bem como em outras áreas [1].

Um exemplo clássico de meio participante é a atmosfera terrestre a qual exerce um efeito atenuante da radiação solar. Outros exemplos de meios participantes são os produtos de combustão de motores - foguetes e proteções térmicas para veículos espaciais [7], entre outras.

$\mathrm{Na}$ modelagem matemática do problema direto de transferência radiativa em um meio participante se faz uso da equação linear de Boltzmann ou Equação de Transferência Radiativa (Radiative Transfer Equation - RTE) a qual resulta da aplicação do princípio da conservação da energia em um meio. Os problemas inversos podem ser formulados matematicamente de modo explicito ou implícito [9], e diversas técnicas têm sido desenvolvidas para a solução dos mesmos $[10,3,11,2]$.

No presente trabalho é proposto a estimativa da espessura óptica, o albedo de espalhamento simples e os coeficientes de reflectividades difusas nas paredes internas de um meio participante unidimensional utilizando para a solução do problema inverso de transferência radiativa através de um sistema neuro-fuzzy combinado com um método de análise de sensibilidade. 


\section{Formulação matemática e Solução do Problema Direto}

O problema direto na transferência radiativa em um meio participante consiste em calcular os valores de intensidade radiativa em qualquer posição do meio e em qualquer direção angular, quando as propriedades do meio, o termo fonte e as condições de contorno são conhecidos.

Considere o problema de transporte de radiação em um meio participante unidimensional, homogêneo (os coeficientes de absorção e de espalhamento não dependem da posição), sem fontes internas (o termo de emissão é considerado desprezível frente à radiação externa incidente no meio), espalhador isotrópico, cinza (as propriedades radiativas do meio independem do comprimento de onda da radiação) e que está sujeito à incidência de radiação isotrópica.

A situação física está representado esquematicamente na Figura 1, onde um meio unidimensional homogêneo de espessura óptica $\tau_{0}$ (sem dependência espectral), espalhador isotrópico, com superfícies refletoras difusas, está sujeito à incidência de radiação externa em suas superfícies de contorno.

Quando as condições de contorno e as propriedades do material são conhecidas, o problema direto de transferência radiativa pode ser resolvido através do fornecimento dos valores da intensidade de radiação em toda posição e direção angular no meio.

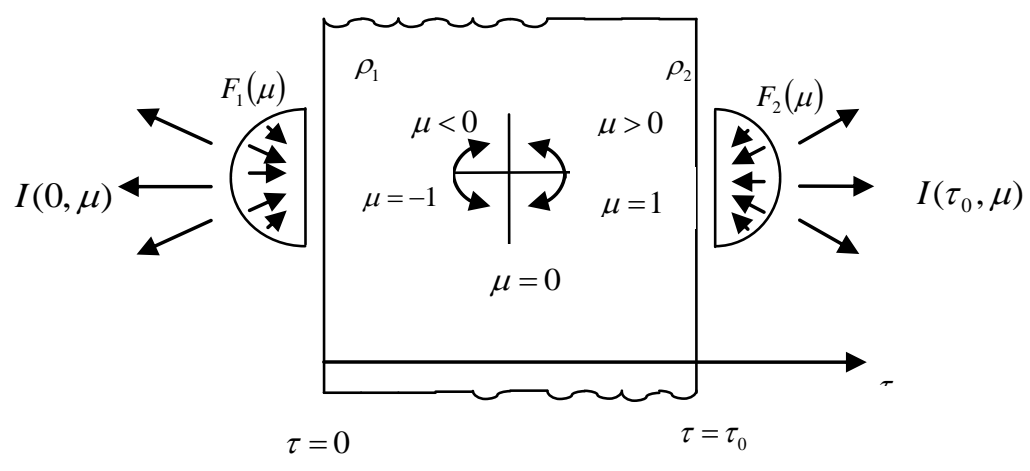

Figura 1 - Radiação isotrópica incidente em um meio participante.

Para o caso de simetria azimutal e desconsiderando o termo de emissão, a equação linearizada de Boltzmann é escrita como

$$
\mu \frac{\partial I(\tau, \mu)}{\partial \tau}+I(\tau, \mu)=\frac{\omega}{2} \int_{-1}^{1} p\left(\mu, \mu^{\prime}\right) I\left(\tau, \mu^{\prime}\right) d \mu^{\prime} \text { em } 0<\tau<\tau_{0},-1 \leq \mu \leq 1
$$

com as seguintes condições de contorno

$$
\begin{aligned}
& I(0, \mu)=F_{1}(\mu)+2 \rho_{1} \int_{0}^{1} I\left(0,-\mu^{\prime}\right) \mu^{\prime} d \mu^{\prime}, \mu>0 \\
& I\left(\tau_{0}, \mu\right)=F_{2}(\mu)+2 \rho_{2} \int_{0}^{1} I\left(\tau_{0}, \mu^{\prime}\right) \mu^{\prime} d \mu^{\prime}, \mu<0
\end{aligned}
$$

onde $p$ é a função de fase de espalhamento anisotrópico, $I(\tau, \mu)$ a intensidade da radiação eletromagnética, $\tau$ a variável óptica, $\tau_{0}$ a espessura óptica do meio, $\mu$ o cosseno do ângulo polar, ou seja, o cosseno do ângulo do feixe de radiação com o eixo $\tau, \omega$ o albedo de espalhamento simples, $\rho_{1}$ e $\rho_{2}$ refletividades difusas das paredes internas do meio e $F_{1}(\mu)$ e $F_{2}(\mu)$ as intensidades das fontes isotrópicas externas da radiação, incidentes respectivamente nas superfícies $\tau=0$ e $\tau=\tau_{0}$.

Se a geometria, as condições de contorno e as propriedades radiantes do meio forem conhecidas, o problema descrito pela Eq. (1), pode ser resolvido diretamente, obtendo-se a intensidade da radiação $I(\tau, \mu)$ para todo $0 \leq \tau \leq \tau_{0}$ e para todo $-1 \leq \mu \leq 1$.

O problema modelado pela Eq. (1) é resolvido utilizando-se o método das ordenadas discretas de Chandrasekhar, no qual o domínio do ângulo polar é discretizado e a integral no lado direito da Eq.(1) é substituída por uma quadratura de Gauss. 
Para os termos no lado esquerdo da Eq. (1) é utilizada uma aproximação por diferenças finitas, e realizando passos para frente e para trás, de $\tau=0$ a $\tau=\tau_{0}$ e de $\tau=\tau_{0}$ a $\tau=0$, respectivamente, obtém-se o valor da intensidade da radiação $I(\tau, \mu)$ para todo $\tau$ e todo $\mu$ do domínio computacional discretizado.

\section{Modelagem do Problema Inverso em Transferência Radiativa}

Segundo o pesquisador russo na área de problemas inversos (PI) Oleg Mikailivitch Alifanov, "a solução de um problema inverso consiste em determinar as causas baseada na observação dos seus efeitos" [3].

Neste trabalho tem-se um problema relacionado à estimativa de quatro parâmetros (constantes) em um problema modelado por uma equação integro-diferencial.

Conhecendo-se as medidas da intensidade da radiação que deixam o meio participante para os diferentes ângulos polares $\theta_{i}$, i.e., $Y_{i}, i=1,2, \cdots, N_{e}$, onde $N_{e}$ é o número total de medidas experimentais, e as propriedades radiativas do meio $Z=\left\{\omega, \tau_{0}, \rho_{1}, \rho_{2}\right\}^{T}$ sendo desconhecidas, procura-se a solução do problema inverso, a partir dos valores das intensidades da radiação que deixa o meio em estudo.

Por conveniência na formulação do problema inverso é aqui considerado que o número de dados experimentais coincide com o número de direções usado na discretização do domínio angular, e, além disso, $Y_{\mathrm{i}}$ é medido no ângulo polar correspondente a $\mu_{\mathrm{i}}$, onde $\mu_{i}=\cos \theta$.

Várias formulações podem ser utilizadas para resolver o problema inverso em transferência radiativa, sendo principalmente objeto, onde um problema de otimização é solucionado com a minimização de uma função custo. Para isso, métodos determinísticos, estocásticos e híbridos têm sido utilizados para minimizar a função de resíduos quadrados entre as intensidades calculadas e as medidas da radiação que deixa o meio, sendo então determinadas com este procedimento as propriedades radiativas requeridas.

Como os dados experimentais não estão disponíveis, é construído um conjunto de dados sintéticos, através de uma solução computacional baseada no problema direto.

Assim, o vetor de incógnitas $Z$, onde

$$
Z=\left\{\omega, \tau_{0}, \rho_{1}, \rho_{2}\right\}^{T}
$$

é determinado a partir das intensidades de radiação

$$
Y_{i}=I_{\text {exp }_{i}}=I_{\text {calc }_{i}}\left(Z_{\text {exact }}\right)+\sigma r_{i}, i=1,2, \cdots, N_{e}
$$

onde $I_{\text {calc }_{i}}$ representa os valores calculados da intensidade de radiação usando os valores exatos dos parâmetros, que numa aplicação real não estão disponíveis, os quais se procura determinar com a solução do problema inverso, $\sigma$ simula o desvio padrão dos erros dos dados experimentais, e $r_{i}$ é um número pseudo-randômico gerado no intervalo [-1,1].

Utilizando os dados experimentais sintéticos $I_{\exp _{i}}$ serão apresentadas nas próximas seções as estimativas para as reflectividades difusas, a espessura óptica e o albedo de espalhamento simples, como solução para o problema inverso de transferência radiativa através de sistemas neuro-fuzzy combinado com um método de análise de sensibilidade.

\section{Análise da Sensibilidade}

A análise de sensibilidade desempenha um papel importante em vários aspectos relacionados com a formulação e solução de problemas inversos [2, 6]. Quando se estuda um problema direto, deseja-se que o mesmo seja pouco sensível aos parâmetros de entrada. Assim, caso ocorra um erro de avaliação nos dados, o fenômeno ainda será bem representado. Na solução de um problema inverso, necessita-se justamente o contrário, pois a maior sensibilidade permitirá a estimativa do parâmetro estudado [5].

$\mathrm{O}$ estudo de sensibilidade consiste em avaliar, numérica ou analiticamente, as derivadas do valor calculado para a grandeza que pode ser observada (medida experimentalmente) com relação aos parâmetros que se deseja estudar. Essas derivadas correspondem à sensibilidade do fenômeno a pequenas variações nos parâmetros de entrada.

Quando se busca estimar diversos parâmetros, os efeitos de cada um deles na intensidade de radiação devem ser diferentes, ou seja, independentes. Se os coeficientes de sensibilidade 
relativos a dois parâmetros diferentes têm a mesma forma, significa que eles afetam a intensidade de radiação da mesma maneira, sendo difícil distinguir suas influências e consequentemente estimá-los.

Para complementar o estudo para a solução deste problema inverso, foi inserida a análise da sensibilidade dos dados experimentais às incógnitas que se deseja determinar $\tau_{0}, \omega, \rho_{1}$ e $\rho_{2}$ por uma aproximação por diferenças finitas (MDF), procurando distinguir a influência destes parâmetros no fenômeno estudado.

\section{Sistemas Neuro-fuzzy}

A elaboração de sistemas com habilidade de usar conhecimento para desempenhar tarefas ou resolver problemas complexos, que se assemelham a problemas reais, é o ponto-chave para desenvolver sistemas inteligentes. Algumas técnicas no desenvolvimento destes sistemas possuem características computacionais particulares que resolvem apenas um conjunto específico de problemas. As redes neurais artificiais e os sistemas fuzzy, por exemplo, não fogem a esta característica. Enquanto as redes neurais são ótimas para a detecção de padrões, elas não são eficientes para explicar como estes padrões são alcançados. Já os sistemas fuzzy que trabalham com a imprecisão e explicam bem a sua tomada de decisão não podem gerar automaticamente as regras que os fizeram tomar aquela decisão.

Sendo assim, diferentes sistemas inteligentes híbridos têm sido desenvolvidos, onde duas ou mais técnicas são combinadas para suprir as suas limitações individuais.

As redes neurais e os sistemas fuzzy propiciam a integração desta tecnologia, denominado de sistemas neuro-fuzzy. Com o objetivo principal de combinar sistemas fuzzy para representar e processar o conhecimento de forma clara e de fácil interpretação, e que aproveitem a capacidade de aprendizado das redes neurais.

As redes neurais são uma boa forma de ajustar os conhecimentos especialistas e gerar automaticamente novas regras fuzzy e novas funções de pertinência. Por outro lado, a lógica fuzzy aperfeiçoa a capacidade de generalização dos sistemas de redes neurais, promovendo uma saída mais realista quando é necessária uma extrapolação além dos limites dos dados do treinamento.

Um sistema neuro-fuzzy (SNF) consiste em vários componentes de sistemas fuzzy tradicionais, exceto pelo fato de que cada estágio é composto por uma camada de neurônios e a potencialidade de aprendizagem da rede neural é utilizada para a obtenção de conhecimento do sistema.

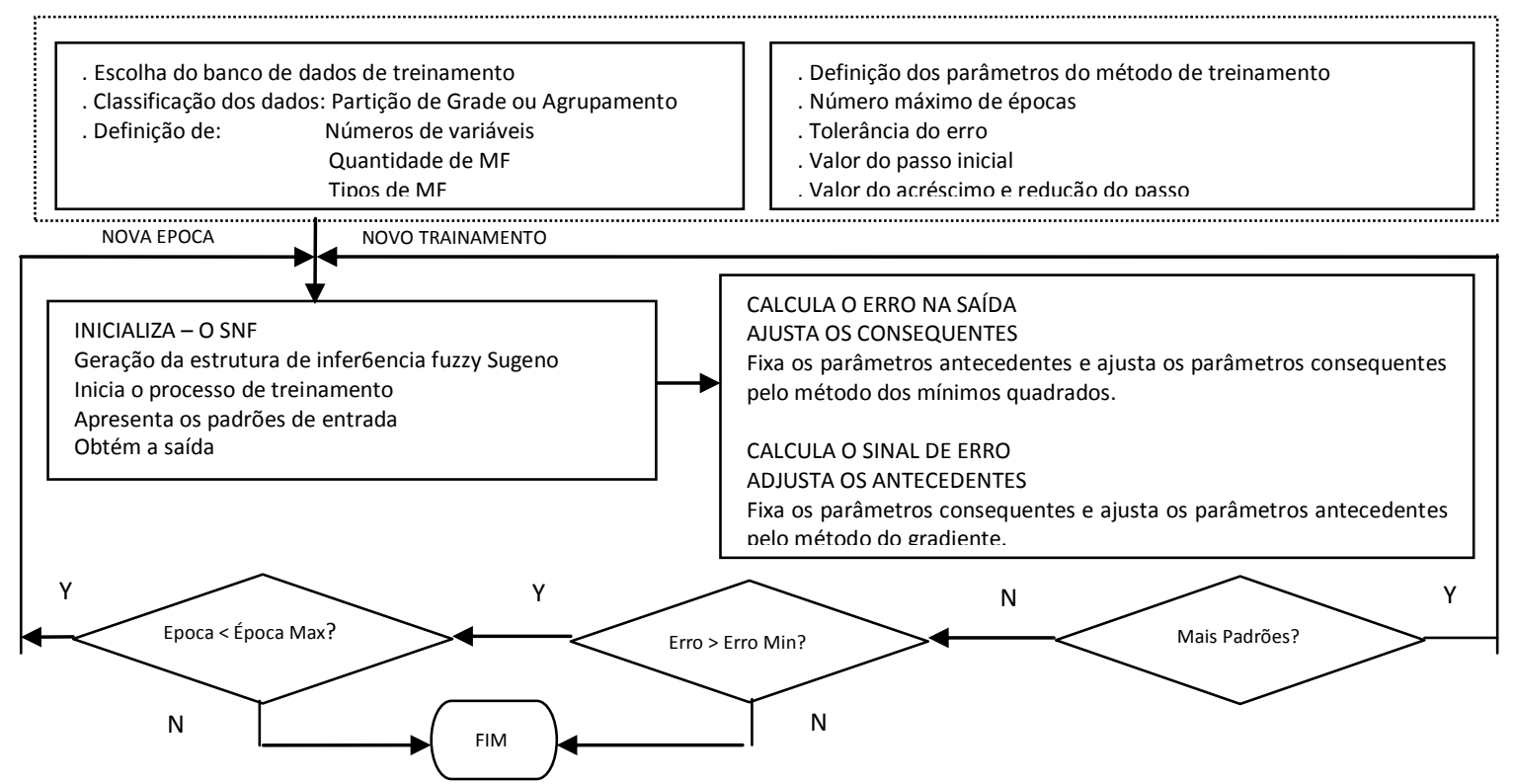

Figura 2: Diagrama em blocos do algoritmo de treinamento do SNF 


\section{Resultados e Discussões}

A Figura 2 representa a fase de treinamento da rede neuro-fuzzy, procurando aumentar o desempenho do sistema pode-se usar um aprendizado híbrido, combinando o método dos mínimos quadrados com o método do gradiente descendente. Para tal é necessário realizar duas fases: uma direta e uma reversa [4].

$\mathrm{Na}$ fase direta o sinal de entrada vai se propagando camada por camada até atingir a camada onde os parâmetros dos consequentes da regra são identificados pelo método dos mínimos quadrados.

Na fase reversa o sinal de erro é retro propagado e os parâmetros dos antecedentes são atualizados pelo método do gradiente descendente.

Para a solução do problema inverso de transferência radiativa de um meio participante unidimensional homogêneo, utilizando as medidas de intensidade de radiação que deixam o meio participante. A modelagem do problema (direto e inverso) e a formulação matemática encontram-se detalhadas nas seções 2 e 3 .

Assim, de forma resumida, o problema direto (PD) consiste em sendo conhecidas a geometria do meio, as propriedades radiativas e as condições limites, determinar a intensidade da radiação que deixa o meio $I(\tau, \mu)$,onde $\tau$ representa a variável óptica espacial (limites $\tau=0$ e $\left.\tau=\tau_{0}\right)$ e $\mu=\cos \theta$, sendo $\theta$ o ângulo polar entre a direção do feixe de radiação e o eixo $\tau$. Nesse caso, representam as reflectividades $\rho_{1}$ e $\rho_{2}$ nos limites $\tau=0$ e $\tau=\tau_{0}$, respectivamente.

Supondo-se que as propriedades radiativas do meio sejam desconhecidas, o que consiste em uma restrição severa para aplicação do $\mathrm{PD}$, mas que existam dados experimentais disponíveis de intensidades de radiação que deixam o meio participante nos limites $\tau=0$ e $\tau=\tau_{0}$, e que o número de dados experimentais seja igual ao número de direções $N_{e}$ usadas na discretização do domínio do ângulo polar $\theta$, empregando o método descrito na seção 2 através de ordenadas discretas com quadratura Gauss-Legendre e com uma aproximação por diferenças finitas para a discretização do domínio espacial, de modo que as intensidades sejam medidas para cada correspondente valor de $\mu=\cos \theta$.

Para validar a solução do problema inverso de transferência radiativa através do sistema neuro-fuzzy em conjunto com um método de análise de sensibilidade (SNF-SA) nos dados experimentais. Serão comparados os resultados obtidos neste método híbrido com os resultados obtidos por dois outros métodos, proposto pela primeira vez por [12] que apresentou uma solução com um método híbrido de Redes Neurais Artificiais com um método de LevenbergMarquardt (RNA-LM), e uma segunda proposta [8] utilizando uma solução com um método de otimização utilizando o funcional de Tikhonov (RT), ressaltando que método de otimização sozinho não converge para a solução esperada.

Para cada parâmetro definido na Tabela 1 foi realizada uma análise de sensibilidade para avaliar as direções que são relevantes para a solução do problema inverso. Procurando apresentar uma melhor visualização dos resultados desta análise, são mostrados na Figura 3 os coeficientes de sensibilidade para cada uma das direções dos dados obtidos experimentalmente $Y_{i}, i=1,2 \ldots, 20$, com o ângulo polar correspondente a $\mu_{i}$ onde $\mu_{i}=\cos \theta$.

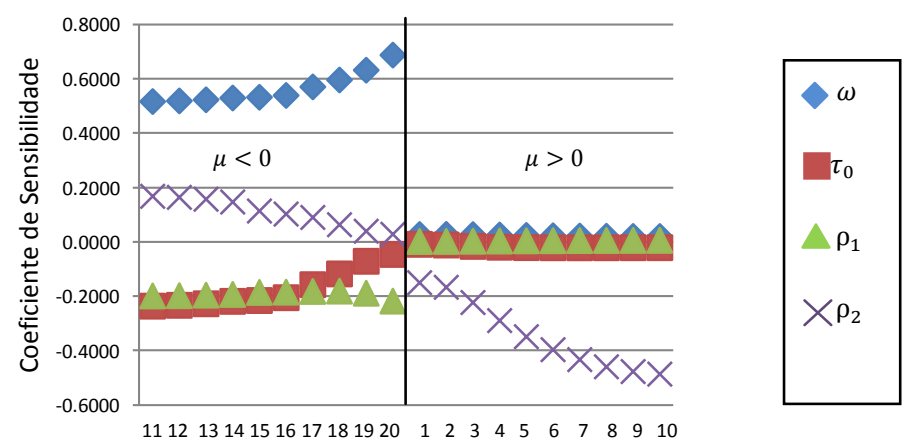

Figura 3: Sensibilidade para o albedo de espalhamento simples $\omega$, a espessura ótica do meio $\tau_{0}$ e as reflectividade difusa $\rho_{1}$ e $\rho_{2}$. 


\begin{tabular}{r|r|c|c}
\hline$\omega$ & $\tau_{0}$ & $\rho_{1}$ & $\rho_{2}$ \\
\hline 0,5 & 1,0 & 0,1 & 0,95 \\
\hline
\end{tabular}

Tabela 1: Valores dos parâmetros a serem estimados, obtidos pelo do método direto $[12,8]$

A partir da análise de sensibilidade serão utilizadas as direções das intensidades para cada um dos casos conforme a Tabela 2.

\begin{tabular}{c|c|c|c}
\hline$\omega$ & $\tau_{0}$ & $\rho_{1}$ & $\rho_{2}$ \\
\hline$\mu_{15} \mathrm{a} \mu_{20}$ & $\mu_{11} \mathrm{a} \mu_{16}$ & $\mu_{15} \mathrm{a} \mu_{20}$ & $\mu_{5} \mathrm{a} \mu_{10}$ \\
\hline
\end{tabular}

Tabela 2: Direções $(\mu)$ das intensidades de radiação com os maiores coeficientes de sensibilidade

Dessa forma, procurando avaliar a eficácia dos métodos SNF, os resultados obtidos através deste, serão comparados aos resultados encontrados pelos métodos RNA-LM e RT, Tabela 3.

\begin{tabular}{c|c|c|c|c|c|c|c|c} 
& \multicolumn{6}{c}{ Ruído presente nos dados experimentais } \\
\cline { 2 - 9 } & \multicolumn{3}{c|}{ RNA-LM } & \multicolumn{4}{c}{ RT } \\
\cline { 2 - 9 } & Valor & Erro \% & Valor & Erro \% & Valor & Erro \% & Valor & Erro \% \\
\hline$\omega$ & 0,502 & 0,40 & 0,502 & 0,40 & 0,5017 & 0,34 & 0,4884 & 2,32 \\
\hline$\tau_{0}$ & 0,999 & 0,10 & 0,999 & 0,10 & 0,9983 & 0,17 & 0,989 & 1,10 \\
\hline$\rho_{1}$ & 0,106 & 6,00 & 0,107 & 7,00 & 0,1015 & 1,50 & 0,0833 & 16,71 \\
\hline$\rho_{2}$ & 0,95 & 0,00 & 0,951 & 0,11 & 0,9496 & 0,04 & 0,9538 & 0,40 \\
\hline
\end{tabular}

Tabela 3: Resultados obtidos pelos métodos RNA-LM [11] e RT [12]

Simulando os dados experimentais, foi utilizado o método descrito na secção 3 , com a inclusão de ruído pseudo-aleatório com erros de 2\%, 3\%, 5\% e 7\% dos valores das intensidades de radiação que saem do meio. Os resultados das estimativas das propriedades radiativas através do método SNF-SA são mostrados na Tabela 4

Analisando os resultados obtidos através do SNF-SA (Tabela 4) em comparação com os resultados nos dois outros métodos utilizados para a comparação na solução do mesmo problema, pode se perceber que em $90 \%$ dos resultados do SNF-SA, este apresentou menores valores de erro percentual, mesmo com a presença de ruído nos dados experimentais.

\begin{tabular}{c|c|c|c|c|c|c|c|c} 
& \multicolumn{9}{c}{ Ruído presente nos dados experimentais } \\
\cline { 2 - 9 } & \multicolumn{2}{c}{ $\pm 0 \%$} & \multicolumn{2}{c}{ $\pm 3 \%$} & \multicolumn{2}{c}{ $\pm 5 \%$} & \multicolumn{2}{c}{ $\pm 7 \%$} \\
\cline { 2 - 9 } & Valor & Erro \% & Valor & Erro \% & Valor & Erro \% & Valor & Erro \% \\
\hline$\omega$ & 0,5000 & 0,00 & 0,4988 & 0,25 & 0,5008 & 0,16 & 0,5005 & 0,10 \\
\hline$\tau_{0}$ & 1,0000 & 0,00 & 1,0007 & 0,07 & 1,0023 & 0,23 & 0,9938 & 0,62 \\
\hline$\rho_{1}$ & 0,1000 & 0,01 & 0,0968 & 3,21 & 0,0971 & 2,86 & 0,1011 & 1,14 \\
\hline$\rho_{2}$ & 0,9500 & 0,00 & 0,9500 & 0,00 & 0,9498 & 0,02 & 0,9504 & 0,04 \\
\hline \multicolumn{7}{c}{ Tabela 4: Resultados obtidos pelo SNF-SA }
\end{tabular}

\section{Conclusões}

Os resultados obtidos com o conjunto de dados experimentais pelo sistema neuro-fuzzy utilizando uma combinação com um método de análise de sensibilidade foram satisfatórios, conforme a comparação apresentada tanto com o método híbrido de Redes Neurais Artificiais com um método de Levenberg-Marquardt, como também utilizando uma solução com um método de otimização utilizando o funcional de Tikhonov. Um outro ponto positivo é a exploração de uma importante característica deste método que é a velocidade de resposta, 
porque, uma vez realizado o treinamento do SNF o resultado de uma nova amostra é um produto simples dos pesos das redes. Ao contrário de outros métodos na solução de problemas inversos, nos quais uma nova amostra acarreta uma grande quantidade de avaliações do problema direto e um grande esforço computacional.

\section{Agradecimentos}

Os autores agradecem à Coordenação de Aperfeiçoamento de Pessoal de Nível Superior (CAPES), ao CNPq, Conselho Nacional de Desenvolvimento Científico e Tecnológico, e da FAPERJ, Fundação Carlos Chagas Filho de Amparo à Pesquisa do Estado do Rio de Janeiro.

pelo apoio financeiro que permitiram a realização deste trabalho.

\section{Referências}

[1] Alvarez Acevedo, N.I.; Roberty, N.C. e Silva Neto, A.J., A One-Dimensional Inverse Radiative Transfer Problem with Time-dependent Boundary Conditions. 4th International Conference on Inverse Problems in Engineering: Theory and Practice, Proc. Angra dos Reis, Brasil, 2002.

[2] Beck, J.V., Blackwell, B. e St. Clair Jr., C.R., Inverse Heat Conduction - Ill-Posed Problems, John Wiley \& Sons, New York, 1985.

[3] Campos Velho, H.F., Problemas Inversos em Pesquisa Espacial, SBMAC, São José dos Campos, SP, 2008.

[4] Jang, J.S.R., Sun, C.T. e Mizutani, E., 1997, Neuro-Fuzzy and Soft Computing: a Computational Approach to Learning and Machine Intelligence, Prentice Hall, New Jersey.

[5] Lugon, J., Pinheiro, R.P.F., Vasconcellos, J.F.V. e Silva Neto, A.J., Estudo de Sensibilidade Aplicado ao Problema Simultâneo de Difusão de Calor e Massa, in Anais do XXV Congresso Nacional de Matemática Aplicada e Computação Científica, 2002.

[6] Milandri, A., Asllanaj, F. and Jeandel, G., Determination of Radiative Properties of Fibrous Media by an Inverse Method - Comparison with the Mie Theory, Journal of Quantitative Spectroscopy \& Radiative Transfer, Vol. 74, pp. 637-653, 2002.

[7] Pessoa Filho, J., Transferência de Calor em Meios Participantes. Série de Palestras de Modelagem Matemática \& Métodos Numéricos, IME-USP, 1998.

[8] Pinheiro, R.P.F., Silva Neto, A.J. e Roberty, N.C., On the use of Bregman Distances for the Solution of Inverse Radiative Transfer Problems in One-Dimensional Participating Media, 5th International Conference on Inverse Problems in Engineering: Theory and Practice, Cambridge, UK, 2005.

[9] Silva Neto, A.J., Explicit and Implicit Formulations for Inverse Radiative Transfer problems. In: 5th World Congress on Computational Mechanics, Mini-Symposium MS 125 - Computational Treatment of Inverse Problems in Mechanics, Vienna, Austria, 2002.

[10] Silva Neto, A.J. e Becceneri, J.C., Técnicas de Inteligência Computacional Inspiradas na Natureza - Aplicação em Problemas Inversos em Transferência Radiativa, SBMAC, 2009.

[11] Silva Neto, A.J. e Moura Neto, F.D., Problemas Inversos: Conceitos Fundamentais e Aplicações, Ed. da Universidade do Rio de Janeiro, Rio de Janeiro, 2005.

[12] Soeiro, F.J.C.P., Soares, P.O. e Silva Neto, A.J., Solution of Inverse Radiative Transfer Problems with Artificial Neural Networks and Hybrid Methods, 13th Inverse Problems in Engineering Seminar, pp. 163-169, Cincinnati, USA, 2004. 